\title{
Algebraic duals of some sets of difference sequences defined by Orlicz functions
}

\author{
Hemen Dutta ${ }^{1}$ and lqbal H Jebril ${ }^{*}$
}

*Correspondence:

iqbal501@hotmail.com

2 Department of Mathematics,

Taibah University, Medina, Saudi

Arabia

Full list of author information is

available at the end of the article

\begin{abstract}
In this paper, we first give a description of some sets of sequences generated by difference operators and defined by Orlicz functions. Then their algebraic duals such as the $\alpha-, \beta-, \gamma$ - and null-duals are computed.
\end{abstract}

MSC: 46A45; 47N40; 65J99; 46A20

Keywords: difference sequences; Orlicz function; algebraic duals

\section{Introduction}

Let $w$ denote the space of all scalar sequences, and any subspace of $w$ is called a sequence space. Let $\ell_{\infty}, c$ and $c_{0}$ be the linear spaces of bounded, convergent and null sequences $x=\left(x_{k}\right)$ with complex terms, respectively, normed by $\|x\|_{\infty}=\sup _{k}\left|x_{k}\right|$, where $k \in N=$ $\{1,2, \ldots\}$, the set of positive integers.

Lindenstrauss and Tzafriri [1] used the Orlicz function and introduced the sequence space $\ell_{M}$ as follows:

$$
\ell_{M}=\left\{\left(x_{k}\right) \in w: \sum_{k=1}^{\infty} M\left(\frac{\left|x_{k}\right|}{\rho}\right)<\infty \text { for some } \rho>0\right\} .
$$

They proved that $\ell_{M}$ is a Banach space normed by

$$
\left\|\left(x_{k}\right)\right\|=\inf \left\{\rho>0: \sum_{k=1}^{\infty} M\left(\frac{\left|x_{k}\right|}{\rho}\right) \leq 1\right\} .
$$

Throughout this section $X$ will denote one of the sequence spaces $\ell_{\infty}, c$ and $c_{0}$.

The notion of difference sequence spaces was introduced by Kizmaz [2]. For some other works on difference sequences, Orlicz functions and related literature, we refer to [3-8]. Let $v=\left(v_{k}\right)$ be any fixed sequence of non-zero complex numbers. Et and Esi [9] generalized the above sequence spaces to the following sequence spaces:

$$
X\left(\Delta_{v}^{m}\right)=\left\{x=\left(x_{k}\right):\left(\Delta_{v}^{m} x_{k}\right) \in X\right\}
$$

for $X=\ell_{\infty}, c$ and $c_{0}$.

In this paper, for an Orlicz function $M$, we can have the following spaces in the line of the spaces studied by Mursaleen et al. [10]:

$$
X\left(M, \Delta_{v}^{m}\right)=\left\{x=\left(x_{k}\right):\left(\Delta_{v}^{m} x_{k}\right) \in X(M)\right\} .
$$


In fact, we get the following spaces:

$$
\begin{aligned}
& \ell_{\infty}\left(M, \Delta_{v}^{m}\right)=\left\{x=\left(x_{k}\right): \sup _{k} M\left(\frac{\left|\Delta_{v}^{m} x_{k}\right|}{\rho}\right)<\infty \text { for some } \rho>0\right\}, \\
& c\left(M, \Delta_{v}^{m}\right)=\left\{x=\left(x_{k}\right): \lim _{k \rightarrow \infty} M\left(\frac{\left|\Delta_{v}^{m} x_{k}-L\right|}{\rho}\right)=0 \text { for some } L \text { and } \rho>0\right\}, \\
& c_{0}\left(M, \Delta_{v}^{m}\right)=\left\{x=\left(x_{k}\right): \lim _{k \rightarrow \infty} M\left(\frac{\left|\Delta_{v}^{m} x_{k}\right|}{\rho}\right)=0 \text { for some } \rho>0\right\},
\end{aligned}
$$

where $\Delta_{v}^{m} x_{k}=\Delta_{v}^{m-1} x_{k}-\Delta_{v}^{m-1} x_{k+1}, \Delta_{v}^{m} x_{k}=\sum_{i=0}^{m}(-1)^{i}\left(\begin{array}{c}m \\ i\end{array}\right) v_{k+i} x_{k+i}$ for all $k \in N$.

Bektaş et al. [11] introduced the difference operator $\Delta_{v}^{(m)}$ and defined it as follows:

$$
\Delta_{v}^{(m)} x_{k}=\sum_{i=0}^{m}(-1)^{i}\left(\begin{array}{c}
m \\
i
\end{array}\right) v_{k-i} x_{k-i} \quad \text { for all } k \in N
$$

Using this difference operator, we can construct the following sequence space:

$$
X\left(M, \Delta_{v}^{(m)}\right)=\left\{x=\left(x_{k}\right):\left(\Delta_{v}^{(m)} x_{k}\right) \in X(M)\right\} .
$$

The operator

$$
\Sigma^{(m)}: w \rightarrow w
$$

is defined by

$$
\Sigma^{(1)} x_{k}=\sum_{j=0}^{k} x_{j} \quad(k=0,1, \ldots), \quad \Sigma^{(m)}=\Sigma^{(1)} o \Sigma^{(m-1)} \quad(m \geq 2)
$$

and

$$
\Sigma^{(m)} o \Delta^{(m)}=\Delta^{(m)} o \Sigma^{(m)}=\mathrm{id}, \quad \text { the identity on } w(\text { see }[9,12])
$$

Now, for subsequent use, we slightly generalize the above definition as follows.

We define

$$
\Sigma_{v}^{(m)}: w \rightarrow w
$$

by

$$
\Sigma_{v}^{(1)} x_{k}=\sum_{j=0}^{k} v_{j} x_{j} \quad(k=0,1, \ldots), \quad \Sigma_{v}^{(m)}=\Sigma_{v}^{(m)} o \Sigma_{v}^{(m-1)} \quad(m \geq 2)
$$

and

$$
v^{-1} \Sigma_{v}^{(m)} o \Delta_{v}^{(m)}=v^{-1} \Delta_{v}^{(m)} o \Sigma_{v}^{(m)}=\mathrm{id}, \quad \text { the identity on } w \text { and } v^{-1}=\left(v_{k}^{-1}\right) .
$$


Now, for $x \in X\left(M, \Delta_{v}^{m}\right)$, let us define

$$
\|x\|_{\Delta}=\sum_{i=1}^{m}\left|v_{i} x_{i}\right|+\inf \left\{\rho>0: \sup _{k} M\left(\frac{\left|\Delta_{v}^{m} x_{k}\right|}{\rho}\right) \leq 1\right\} .
$$

It can be shown that $\left(X\left(M, \Delta_{v}^{m}\right),\|\cdot\|_{\Delta}\right)$ is a $B K$-space.

Again for $x \in X\left(M, \Delta_{v}^{(m)}\right)$, let us define

$$
\|x\|_{\Delta^{\prime}}=\inf \left\{\rho>0: \sup _{k} M\left(\frac{\left|\Delta_{v}^{(m)} x_{k}\right|}{\rho}\right) \leq 1\right\} .
$$

It can be shown that $\left(X\left(M, \Delta_{v}^{(m)}\right),\|\cdot\|_{\Delta^{\prime}}\right)$ is a $B K$-space.

It is trivial that $\left(\Delta_{v}^{m} x_{k}\right) \in X(M)$ if and only if $\left(\Delta_{v}^{(m)} x_{k}\right) \in X(M)$. Also the norms $\|\cdot\|_{\Delta}$ and $\|\cdot\|_{\Delta^{\prime}}$ are equivalent.

Let us define the operator

$$
D: X\left(M, \Delta_{v}^{m}\right) \rightarrow X\left(M, \Delta_{v}^{m}\right)
$$

by $D x=\left(0,0, \ldots, x_{m+1}, x_{m+2}, \ldots\right)$, where $x=\left(x_{1}, x_{2}, \ldots, x_{m}, \ldots\right)$. It is trivial that $D$ is a bounded linear operator on $X\left(M, \Delta_{v}^{m}\right), X=\ell_{\infty}, c$ and $c_{0}$. Furthermore, the set

$$
D\left[X\left(M, \Delta_{v}^{m}\right)\right]=D X\left(M, \Delta_{v}^{m}\right)=\left\{x=\left(x_{k}\right): x \in X\left(M, \Delta_{v}^{m}\right), x_{1}=x_{2}=\cdots=x_{m}=0\right\}
$$

is a subspace of $X\left(M, \Delta_{v}^{m}\right)$ and normed by

$$
\|x\|_{\Delta}=\inf \left\{\rho>0: \sup _{k} M\left(\frac{\left|\Delta_{v}^{m} x_{k}\right|}{\rho}\right) \leq 1\right\} \quad \text { in } D X\left(M, \Delta_{v}^{m}\right) .
$$

$D X\left(M, \Delta_{v}^{m}\right)$ and $X(M)$ are equivalent as topological spaces since

$$
\Delta_{v}^{m}: D X\left(M, \Delta_{v}^{m}\right) \rightarrow X(M)
$$

defined by

$$
\Delta_{v}^{m} x=y=\left(\Delta_{v}^{m} x_{k}\right)
$$

is a linear homeomorphism.

Moreover, obviously

$$
\begin{array}{ll}
\Delta_{v}^{(m)}: X\left(M, \Delta_{v}^{(m)}\right) \rightarrow X(M), & \Delta_{v}^{(m)} x=y=\left(\Delta_{v}^{(m)} x_{k}\right), \\
\Sigma_{v}^{(m)}: X(M) \rightarrow X\left(M, \Delta_{v}^{(m)}\right), & \Sigma_{v}^{(m)} x=y=\left(\Sigma_{v}^{(m)} x_{k}\right)
\end{array}
$$

are isometric isomorphisms for $X=\ell_{\infty}, c$ and $c_{0}$.

Hence $\ell_{\infty}\left(M, \Delta_{v}^{m}\right), c\left(M, \Delta_{v}^{m}\right)$ and $c_{0}\left(M, \Delta_{v}^{m}\right)$ are isometrically isomorphic to $\ell_{\infty}(M)$, $c(M)$ and $c_{0}(M)$, respectively. 
Moreover, $X\left(M, \Delta_{v}^{i}\right) \subset X\left(M, \Delta_{v}^{m}\right)$ for $i=0,1, \ldots, m-1$, which follows from the following inequality and convexity of $M$ :

$$
M\left(\frac{\left|\Delta_{v}^{m} x_{k}\right|}{2 \rho}\right) \leq \frac{1}{2} M\left(\frac{\left|\Delta_{v}^{m-1} x_{k}\right|}{\rho}\right)+\frac{1}{2} M\left(\frac{\left|\Delta_{v}^{m-1} x_{k+1}\right|}{\rho}\right)
$$

Investigation of spaces is often combined with that of duals. The algebraic dual space is defined for all vector spaces. When defined for a topological vector space, there is a subspace of this dual space, corresponding to continuous linear functionals, which constitutes a continuous dual space. For any finite-dimensional normed vector space or topological vector space, such as Euclidean $n$-space, the continuous dual and the algebraic dual coincide. This is, however, false for any infinite-dimensional normed space. For some related literature on duality relevant to this paper, we refer to $[9,11,13,14]$. Our results of this paper will generalize few existing results as well as generate some new results in the literature of algebraic duality within the field of functional analysis.

\section{Computation of algebraic duals}

In this section we compute the $\alpha-, \beta-, \gamma$ - and $N$-duals of the spaces $\ell_{\infty}\left(M, \Delta_{v}^{m}\right), c\left(M, \Delta_{v}^{m}\right)$ and $c_{0}\left(M, \Delta_{v}^{m}\right)$.

Definition 2.1 [13] Let $X$ be a sequence space and define

$$
\begin{aligned}
& X^{\alpha}=\left\{a=\left(a_{k}\right): \sum_{k=1}^{\infty}\left|a_{k} x_{k}\right|<\infty, \forall x \in X\right\}, \\
& X^{\beta}=\left\{a=\left(a_{k}\right): \sum_{k=1}^{\infty} a_{k} x_{k} \text { is convergent, } \forall x \in X\right\}, \\
& X^{\gamma}=\left\{a=\left(a_{k}\right): \sup _{n}\left|\sum_{k=1}^{n} a_{k} x_{k}\right|<\infty, \forall x \in X\right\}, \\
& X^{N}=\left\{a=\left(a_{k}\right): \lim _{k} a_{k} x_{k}=0, \forall x \in X\right\},
\end{aligned}
$$

then $X^{\alpha}, X^{\beta}, X^{\gamma}$ and $X^{N}$ are called the $\alpha-, \beta-, \gamma$ - and $N$-(or null) duals of $X$, respectively. It is known that if $X \subset Y$, then $Y^{\eta} \subset X^{\eta}$ for $\eta=\alpha, \beta, \gamma$ and $N$.

Lemma 2.1 [2] Let $m$ be a positive integer. Then there exist positive constants $C_{1}$ and $C_{2}$ such that

$$
C_{1} k^{m} \leq\left(\begin{array}{c}
m+k \\
k
\end{array}\right) \leq C_{2} k^{m}, \quad k=0,1,2, \ldots
$$

Lemma $2.2 x \in \ell_{\infty}\left(M, \Delta_{v}^{m}\right)$ implies $\sup _{k} M\left(\frac{\left|k^{-1} \Delta_{v}^{m-1} x_{k}\right|}{\rho}\right)<\infty$ for some $\rho>0$.

Proof Let $x \in \ell_{\infty}\left(M, \Delta_{v}^{m}\right)$, then

$$
\sup _{k} M\left(\frac{\left|\Delta_{v}^{m-1} x_{k}-\Delta_{v}^{m-1} x_{k+1}\right|}{\rho}\right)<\infty \text { for some } \rho>0 .
$$


Then there exists $U>0$ such that

$$
M\left(\frac{\left|\Delta_{v}^{m-1} x_{k}-\Delta_{v}^{m-1} x_{k+1}\right|}{\rho}\right)<U \text { for all } k \in N
$$

Taking $\eta=k \rho$, for an arbitrary fixed positive integer $k$, by the subadditivity of modulus, the monotonicity and convexity of $M$ :

$$
M\left(\frac{\left|\Delta_{v}^{m-1} x_{1}-\Delta_{v}^{m-1} x_{k+1}\right|}{\eta}\right) \leq \frac{1}{k} \sum_{l=1}^{k} M\left(\frac{\left|\Delta_{v}^{m-1} x_{l}-\Delta_{v}^{m-1} x_{l+1}\right|}{\rho}\right)<U
$$

Then the above inequality, the inequality

$$
\frac{\left|\Delta_{v}^{m-1} x_{k+1}\right|}{(k+1) \rho} \leq \frac{1}{k+1}\left(\frac{\left|\Delta_{v}^{m-1} x_{1}\right|}{\rho}+k \frac{\left|\Delta_{v}^{m-1} x_{1}-\Delta_{v}^{m-1} x_{k+1}\right|}{k \rho}\right)
$$

and the convexity of $M$ imply

$$
\begin{aligned}
M\left(\frac{\left|\Delta_{v}^{m-1} x_{k+1}\right|}{(k+1) \rho}\right) & \leq \frac{1}{k+1}\left(M\left(\frac{\left|\Delta_{v}^{m-1} x_{1}\right|}{\rho}\right)+k M\left(\frac{\left|\Delta_{v}^{m-1} x_{1}-\Delta_{v}^{m-1} x_{k+1}\right|}{k \rho}\right)\right) \\
& \leq \max \left\{M\left(\frac{\left|\Delta_{v}^{m-1} x_{1}\right|}{\rho}\right), U\right\}<\infty .
\end{aligned}
$$

Hence we have the desired result.

Hence we have the following lemma.

\section{Lemma 2.3}

(i) $x \in \ell_{\infty}\left(M, \Delta_{v}^{m}\right)$ implies $\sup _{k} M\left(\frac{\left|k^{-m} v_{k} x_{k}\right|}{\rho}\right)<\infty$ for some $\rho>0$,

(ii) $x \in \ell_{\infty}\left(M, \Delta_{v}^{m}\right)$ implies $\sup _{k} k^{-m}\left|v_{k} x_{k}\right|<\infty$.

Theorem 2.4 Let $M$ be an Orlicz function. Then

(i) $\left[c_{0}\left(M, \Delta_{v}^{m}\right)\right]^{\alpha}=\left[c\left(M, \Delta_{v}^{m}\right)\right]^{\alpha}=\left[\ell_{\infty}\left(M, \Delta_{v}^{m}\right)\right]^{\alpha}=D_{1}$,

(ii) $D_{1}^{\alpha}=D_{2}$,

where

$$
\begin{aligned}
& D_{1}=\left\{a=\left(a_{k}\right): \sum_{k=1}^{\infty} k^{m}\left|v_{k}^{-1} a_{k}\right|<\infty\right\}, \\
& D_{2}=\left\{b=\left(b_{k}\right): \sup _{k} k^{-m}\left|v_{k} b_{k}\right|<\infty\right\} .
\end{aligned}
$$

Proof (i) Let $a \in D_{1}$, then $\sum_{k=1}^{\infty} k^{m}\left|v_{k}^{-1} a_{k}\right|<\infty$. Now, for any $x \in \ell_{\infty}\left(M, \Delta_{v}^{m}\right)$, we have $\sup _{k} k^{-m}\left|v_{k} x_{k}\right|<\infty$. Then we have

$$
\sum_{k=1}^{\infty}\left|a_{k} x_{k}\right| \leq \sup _{k} k^{-m}\left|v_{k} x_{k}\right| \sum_{k=1}^{\infty} k^{m}\left|a_{k} v_{k}^{-1}\right|<\infty
$$

Hence $a \in\left[\ell_{\infty}\left(M, \Delta_{v}^{m}\right)\right]^{\alpha}$. 
Conversely, suppose that $a \in\left[X\left(M, \Delta_{v}^{m}\right)\right]^{\alpha}$ for $X=c$ and $\ell_{\infty}$. Then $\sum_{k=1}^{\infty}\left|a_{k} x_{k}\right|<\infty$ for each $x \in X\left(M, \Delta_{v}^{m}\right)$. So we can take

$$
x_{k}=k^{m} v_{k}^{-1}, \quad k \geq 1 .
$$

Then

$$
\sum_{k=1}^{\infty} k^{m}\left|v_{k}^{-1} a_{k}\right|=\sum_{k=1}^{\infty}\left|a_{k} x_{k}\right|<\infty .
$$

This implies that $a \in D_{1}$.

Again suppose that $a \in\left[c_{0}\left(M, \Delta_{v}^{m}\right)\right]^{\alpha}$ and $a \notin D_{1}$. Then there exists a strictly increasing sequence $\left(n_{i}\right)$ of positive integers $n_{i}$ with $n_{1}<n_{2}<\cdots$ such that

$$
\sum_{k=n_{i}+1}^{n_{i+1}} k^{m}\left|v_{k}^{-1} a_{k}\right|>i
$$

Define $x \in c_{0}\left(M, \Delta_{v}^{m}\right)$ by

$$
x_{k}= \begin{cases}0, & 1 \leq k \leq n_{1}, \\ k^{m} v_{k} \operatorname{sgn} a_{k / i}, & n_{i}<k \leq n_{i+1} .\end{cases}
$$

Then we have

$$
\begin{aligned}
\sum_{k=1}^{\infty}\left|a_{k} x_{k}\right| & =\sum_{k=n_{1}+1}^{n_{2}}\left|a_{k} x_{k}\right|+\cdots+\sum_{k=n_{i}+1}^{n_{i+1}}\left|a_{k} x_{k}\right|+\cdots \\
& =\sum_{k=n_{1}+1}^{n_{2}} k^{m}\left|v_{k}^{-1} a_{k}\right|+\cdots+\frac{1}{i} \sum_{k=n_{i}+1}^{n_{i+1}} k^{m}\left|v_{k}^{-1} a_{k}\right|+\cdots \\
& >1+1+\cdots=\infty .
\end{aligned}
$$

This contradicts $a \in\left[c_{0}\left(M, \Delta_{v}^{m}\right)\right]^{\alpha}$. Hence $a \in D_{1}$. This completes the proof of (i).

(ii) The proof is similar to that of part (i).

If we take $v_{k}=1$ for all $k \in N$ in Theorem 2.4, then we obtain the following corollary.

\section{Corollary 2.5 Let $M$ be an Orlicz function. Then}

(i) $\left[c_{0}\left(M, \Delta^{m}\right)\right]^{\alpha}=\left[c\left(M, \Delta^{m}\right)\right]^{\alpha}=\left[\ell_{\infty}\left(M, \Delta^{m}\right)\right]^{\alpha}=E_{1}$,

(ii) $E_{1}^{\alpha}=E_{2}$,

where

$$
\begin{aligned}
& E_{1}=\left\{a=\left(a_{k}\right): \sum_{k=1}^{\infty} k^{m}\left|a_{k}\right|<\infty\right\}, \\
& E_{2}=\left\{b=\left(b_{k}\right): \sup _{k} k^{-m}\left|b_{k}\right|<\infty\right\} .
\end{aligned}
$$


Theorem 2.6 Let $M$ be an Orlicz function. Then $\left[c\left(M, \Delta_{v}^{m}\right)\right]^{N}=\left[\ell_{\infty}\left(M, \Delta_{v}^{m}\right)\right]^{N}=F_{1}$, where

$$
F_{1}=\left\{a=\left(a_{k}\right): \lim _{k} k^{m} v_{k}^{-1} a_{k}=0\right\} .
$$

Proof The proof is immediate using Lemma 2.3(ii).

The following lemma will be used in the next theorem.

Lemma 2.7 [6] Let $\left(p_{n}\right)$ be a sequence of positive numbers increasing monotonically to infinity.

(i) If $\sup _{n}\left|\sum_{v=1}^{n} p_{v} a_{v}\right|<\infty$, then $\sup _{n}\left|p_{n} \sum_{k=n+1}^{\infty} a_{k}\right|<\infty$.

(ii) If $\sum_{k} p_{k} a_{k}$ is convergent, then $\lim _{n} p_{n} \sum_{k=n+1}^{\infty} a_{k}=0$.

Theorem 2.8 Let $M$ be an Orlicz function and $c_{0}^{+}$denote the set of all positive null sequences. Then

(i) $\left[D \ell_{\infty}\left(M, \Delta_{v}^{m}\right)\right]^{\beta}=\left[D c\left(M, \Delta_{v}^{m}\right)\right]^{\beta}=G_{1}$,

(ii) $\left[D c_{0}\left(M, \Delta_{v}^{m}\right)\right]^{\beta}=G_{2}$,

(iii) $\left[D \ell_{\infty}\left(M, \Delta_{v}^{m}\right)\right]^{\gamma}=\left[D c\left(M, \Delta_{v}^{m}\right)\right]^{\gamma}=H_{1}$,

(iv) $\left[D c_{0}\left(M, \Delta_{v}^{m}\right)\right]^{\gamma}=H_{2}$,

where

$$
\begin{aligned}
& G_{1}=\left\{a=\left(a_{k}\right): \sum_{k=1}^{\infty} a_{k} v_{k}^{-1} \sum_{j=1}^{k-m}\left(\begin{array}{c}
k-j-1 \\
m-1
\end{array}\right)\right. \text { is convergent, } \\
& \left.\sum_{k=1}^{\infty}\left|\sum_{j=k+1}^{\infty} v_{j}^{-1} a_{j}\right| \sum_{j=1}^{k-m+1}\left(\begin{array}{c}
k-j-1 \\
m-2
\end{array}\right)<\infty\right\}, \\
& G_{2}=\left\{a=\left(a_{k}\right): \sum_{k=1}^{\infty} a_{k} v_{k}^{-1} \sum_{j=1}^{k-m}\left(\begin{array}{c}
k-j-1 \\
m-1
\end{array}\right) u_{j}\right. \text { converges and } \\
& \left.\sum_{k=1}^{\infty}\left|\sum_{j=k+1}^{\infty} v_{j}^{-1} a_{j}\right| \sum_{j=1}^{k-m+1}\left(\begin{array}{c}
k-j-1 \\
m-2
\end{array}\right) u_{j}<\infty, \forall u \in c_{0}^{+}\right\}, \\
& H_{1}=\left\{a=\left(a_{k}\right): \sup _{n}\left|\sum_{k=1}^{n} a_{k} v_{k}^{-1} \sum_{j=1}^{k-m}\left(\begin{array}{c}
k-j-1 \\
m-1
\end{array}\right)\right|<\infty,\right. \\
& \left.\sum_{k=1}^{\infty}\left|\sum_{j=k+1}^{\infty} v_{j}^{-1} a_{j}\right| \sum_{j=1}^{k-m+1}\left(\begin{array}{c}
k-j-1 \\
m-2
\end{array}\right)<\infty\right\}, \\
& H_{2}=\left\{a=\left(a_{k}\right): \sup _{n}\left|\sum_{k=1}^{n} a_{k} v_{k}^{-1} \sum_{j=1}^{k-m}\left(\begin{array}{c}
k-j-1 \\
m-1
\end{array}\right) u_{j}\right|<\infty\right. \text {, } \\
& \left.\sum_{k=1}^{\infty}\left|\sum_{j=k+1}^{\infty} v_{j}^{-1} a_{j}\right| \sum_{j=1}^{k-m+1}\left(\begin{array}{c}
k-j-1 \\
m-2
\end{array}\right) u_{j}<\infty, \forall u \in c_{0}^{+}\right\} .
\end{aligned}
$$

Proof We give the proof for part (i) for $D \ell_{\infty}\left(M, \Delta_{v}^{m}\right)$, and the proof of other parts follows similarly using Lemma 2.7. For details, one may refer to [11]. 
For each $x \in D \ell_{\infty}\left(M, \Delta_{v}^{m}\right)$, there exists one and only one $y=\left(y_{k}\right) \in \ell_{\infty}(M)$ such that

$$
x_{k}=v_{k}^{-1} \sum_{j=1}^{k-m}\left(\begin{array}{c}
k-j-1 \\
m-1
\end{array}\right) y_{j}, \quad y_{1-m}=y_{2-m}=\cdots=y_{0}=0
$$

for sufficiently large $k$ by (1.1). Let $a \in G_{1}$. Then, using the same technique as applied in $\left[11\right.$, p.429], we can show that $a \in\left[D \ell_{\infty}\left(M, \Delta_{v}^{m}\right)\right]^{\beta}$.

Let $a \in\left[D \ell_{\infty}\left(M, \Delta_{v}^{m}\right)\right]^{\beta}$. Again, using the same technique as applied in [11, pp.429-430], we can show that $a \in G_{1}$.

This completes the proof.

\section{Conclusion}

Although we conclude this paper here, the following further suggestion remains open:

What is the $N$-dual of the space $c_{0}\left(M, \Delta_{v}^{m}\right)$ ?

Competing interests

The authors declare that they have no competing interests.

Authors' contributions

HD carried out the initial description of the spaces. IHJ proposed the structure of the paper. HD and IHJ both formulated and proved the results. All authors read and approved the final manuscript.

\section{Author details}

'Department of Mathematics, Gauhati University, Guwahati, 781014, India. ²Department of Mathematics, Taibah University, Medina, Saudi Arabia.

Received: 29 May 2014 Accepted: 8 July 2014 Published: 19 Aug 2014

\section{References}

1. Lindenstrauss, J, Tzafriri, L: On Orlicz sequence spaces. Isr. J. Math. 10, 379-390 (1971)

2. Kizmaz, H: On certain sequence spaces. Can. Math. Bull. 24(2), 169-176 (1981)

3. Dutta, $\mathrm{H}$ : Characterization of certain matrix classes involving generalized difference summability spaces. Appl. Sci. 11, 60-67 (2009)

4. Dutta, $H$, Bilgin, $T$ : Strongly $\left(V^{\lambda}, A, \Delta_{v m}^{n}, p\right)$-summable sequence spaces defined by an Orlicz function. Appl. Math. Lett. 24(7), 1057-1062 (2011)

5. Dutta, H, Jebril, IH: An extension of modular sequence spaces. Abstr. Appl. Anal. 2013, Article ID 371806 (2013)

6. Et, M: On some topological properties of generalized difference sequence spaces. Int. J. Math. Math. Sci. 24(11), 785-791 (2000)

7. Karakaya, V, Dutta, H: On some vector valued generalized difference modular sequence spaces. Filomat 25(3), 15-27 (2011)

8. Tripathy, BC, Dutta, H: On some lacunary difference sequence spaces defined by a sequence of Orlicz functions and $q$-lacunary $\Delta_{m}^{n}$-statistical convergence. An. Şt. Univ. Ovidius Constanţa 20(1), 417-430 (2012)

9. Et, M, Esi, A: On Köthe-Toeplitz duals of generalized difference sequence spaces. Bull. Malays. Math. Sci. Soc. 23, 25-32 (2000)

10. Mursaleen, M, Khan, A, Qamaruddin: Difference sequence spaces defined by Orlicz functions. Demonstr. Math. 32(1), 145-150 (1999)

11. Bektaş, ÇA, Et, M, Çolak, R: Generalized difference sequence spaces and their dual spaces. J. Math. Anal. Appl. 292 423-432 (2004)

12. Dutta, H, Başar, F: A generalization of Orlicz sequence spaces by Cesàro mean of order one. Acta Math. Univ. Comen. 80(2), 185-200 (2011)

13. Dutta, H: On Köthe-Toeplitz and null duals of some difference sequence spaces defined by Orlicz functions. Eur. J. Pure Appl. Math. 2(4), 554-563 (2009)

14. Malkowsky, E, Parasar, SD: Matrix transformation in spaces of bounded and convergent difference sequences of order m. Analysis 17, 87-97 (1997)

$10.1186 / 1029-242 X-2014-300$

Cite this article as: Dutta and Jebril: Algebraic duals of some sets of difference sequences defined by Orlicz

functions. Journal of Inequalities and Applications 2014, 2014:300 